\title{
Data Exchange Between Immunization Registry and Disease Surveillance System
}

\author{
Hwa-Gan Chang ${ }^{\star 1}$, Griffin Jacqueline ${ }^{2}$, Hans Proske ${ }^{2}$, Megan Meldrum ${ }^{1}$ and Elizabeth \\ Rausch-Phung ${ }^{1}$
}

${ }^{1}$ New York State Department of Health, Albany, NY, USA; ${ }^{2}$ NTT Data, Albany, NY, USA

\section{Objective}

To establish the infrastructure to provide a linkage between the immunization registry and disease surveillance system using standard for data exchange.

\section{Introduction}

New York State Department of Health (NYSDOH) implemented a Communicable Disease Electronic Surveillance System (CDESS), a single and secure application used by 57 local health departments (LHDs), hospital infection control programs and NYSDOH staff to collect, integrate, analyze, and report data for infectious disease surveillance. New York State Immunization Information System (NYSIIS) is a mandated application for providers to report all vaccinations of persons $<19$ years old residing in New York State (excluding New York City). Currently, LHD staff must manually search NYSIIS for vaccine preventable disease case investigations and re-enter the immunization histories into CDESS. NYSIIS has built a HL7 query functionality which can be used to automate the data exchange between NYSIIS and CDESS.

\section{Methods}

The business rules and data specifications for exchange of vaccine histories of reported pertussis cases between NYSIIS and CDESS were developed. A process was established, a daily HL7 query file of reported pertussis cases from CDESS was generated and the file was matched against NYSIIS by patient's last name, first name, date of birth, gender, and address. NYSIIS utilized its process of generating a HL7 response file that contains vaccine history on matched cases to send back to CDESS. CDESS then processes the response file and loads vaccine history into its vaccine table for the matched pertussis cases.

\section{Results}

An automated process was developed and implemented in April 2015. Between April 1 through July 31, 2015, there were 166 pertussis cases reported through CDESS, 32 cases over aged 19 years old were excluded, and $80(60 \%)$ cases were matched in NYSIIS. All pertussis vaccine related information (such as vaccine date, vaccine type, manufacturer, and vaccine lot number) from NYSIIS were populated in CDESS corresponding data fields for matched cases. Fifty-four cases were not found as matches in NYSIIS due to not matching address, no NYSIIS record, or no pertussis vaccine.

\section{Conclusions}

By using existing NYSIIS query functionality, this is an easy process to establish an automated linkage for data exchange between NYSIIS and disease surveillance systems. This process provides a more timely and efficient way to assist LHD staff to get vaccine information for vaccine preventable cases. NYSIIS does not contain all vaccine information (eg. manufacturer, vaccine lot number) and LHD staff may still be required to contact providers.

\section{Keywords}

Surveillance system; Immunization registry; Data exchange

\section{Acknowledgments}

This publication was supported by Cooperative Agreement Number U50CK000423 from the Centers for Disease Control and Prevention (CDC). Its contents are solely the responsibility of the authors and do not necessarily represent the official views of CDC.

\section{${ }^{*}$ Hwa-Gan Chang}

E-mail: hwagan.chang@health.ny.gov 